\title{
Appropriate Hospital Discharge Timing after Laparoscopic Cholecystectomy: Comparison of Postoperative Day 1 vs. Day 2 Discharge Protocol
}

\author{
Jae Woo Park, M.D., Munjin Kim, M.D., Sang Kuon Lee, M.D. \\ Department of Surgery, Daejeon St. Mary's Hospital, College of Medicine, The Catholic University of Korea, Daejeon, Korea
}

Purpose: The critical pathway $(\mathrm{CP})$ was introduced as a means to provide quality healthcare service in many fields of surgery. CP may increase the patient's satisfaction rate and lowering hospital stay and medical cost also. We aimed to compare the two kinds of $\mathrm{CP}$ applied in laparoscopic cholecystectomy patients by different hospital stay length.

Methods: From March 2016 to October 2016, 71 patients were enrolled in this analysis among 241 patients who underwent elective laparoscopic cholecystectomy. Patients were divided into two groups, 38 patients in the 1-day CP group and 33 patients in the 2-day CP group. In a retrospective review, surgical outcomes and related hospital costs were analyzed.

Results: Preoperative characteristics were not different between two CP groups. In analysis of operative outcome, 2-day CP group showed longer operative time than 1-day CP (73.4 vs 54.1 min, $p<0.001)$; otherwise, there was no significant difference in frequency of postop complications (6.1\% vs $2.6 \%, p=0.474)$, numerical rating scale (NRS) pain score (1.82 vs 2.16, $p=0.052$ ), and count of analgesics injection $(0.12$ vs $0.16, p=0.754)$. Total admission cost and actual patient's expenditures were higher in 2-day CP group, but there was no statistically significant difference $\left(347.04\right.$ vs $306.69 \times 10^{4}$ won, $p=0.106 ; 147.85$ vs $125.58 \times 10^{4}$ won, $p=0.276$ ).

Conclusion: The length of hospital stay was shortened in 1-day CP group than in 2-day CP group, while there was no difference in other parameters. Therefore, it is feasible and safe practical policy the use 1-day CP in selected patients who undergo cholecystectomy according to our results.

Keywords: Cholecystectomy, Laparoscopic, Critical pathways, Hospital costs
Received September 1, 2018

Revised 1st September 20, 2018 2nd October 17, 2018

Accepted October 19, 2018

Corresponding author

Sang Kuon Lee

Department of Surgery, Daejeon

St. Mary's Hospital, College of

Medicine, The Catholic University

of Korea, 64 Daeheung-ro, Jung-gu, Daejeon 34943, Korea

Tel: $+82-42-220-9520$

Fax: +82-42-220-9565

E-mail: luislee@catholic.ac.kr ORCID:

https://orcid.org/0000-0002-3720-2461
This is an Open Access article distributed under the terms of the Creative Commons Attribution Non-Commercial License (http:// creativecommons.org/licenses/by-nc/4.0/) which permits unrestricted non-commercial use, distribution, and reproduction in any medium, provided the original work is properly cited.
Copyright $\odot 2019$ The Journal of Minimally Invasive Surgery. All rights reserved.

\section{INTRODUCTION}

Laparoscopic cholecystectomy is the most effective treatment for patients with symptomatic gallbladder diseases. ${ }^{1-3}$ Laparoscopic cholecystectomy has been widely used because it is associated with shorter hospital stay, shorter recovery period, lower hospital cost, and postoperative pain reduction. Open cholecystectomy has been limited to acute cholecys- titis with complications. ${ }^{4}$ In recent years, in many hospitals, laparoscopic cholecystectomy has been performed under a systematic treatment algorithm using the critical pathway (CP), which is a series of standardized processes for the diagnosis or treatment of a defined condition based on practice guidelines in order to improve outcomes and reduce costs. ${ }^{3,5-7}$ Laparoscopic cholecystectomy using CP is based on the concept of rapid recovery after surgery (Enhanced Recovery After Sur- 
gery (ERAS)). ${ }^{8}$ ERAS is a basic concept that helps patients to recover faster after surgery by minimizing the physical effects on the human body and reducing the stress response. ${ }^{9}$

It has been proved in other studies that it is possible to effectively reduce the hospital stay and the cost of hospitalization when CP is applied. Furthermore, some studies revealed that there was no significant difference in the re-admission rate, visual analogue scale (VAS) pain score, and postoperative complication rate comparing day-surgery and overnight stay surgery in laparoscopic cholecystectomy. However, it was difficult to find studies that directly compare two CPs with different hospital stay period.

The purpose of this study was to evaluate the usefulness of standard guideline for laparoscopic cholecystectomy in terms of length of stay and cost. In this study, we tried to find out whether there is significant difference between 1-day CP (discharge from hospital on postoperative day 1) and 2-day $\mathrm{CP}$ (discharge from hospital on postoperative day 2) focused on medical and socioeconomic viewpoints.

\section{MATERIALS AND METHODS}

This retrospective study was approved by the Institutional Review Boards of the institution (DC16RISI0049). At the department of General Surgery of Daejeon St. Mary's hospital, two types of $\mathrm{CP}$ of laparoscopic cholecystectomy were developed and applied to patients by surgeon's decision: 1-day $\mathrm{CP}$ and 2-day CP. Though there are not different statistically, there was a tendency that patients with multiple comorbidities and history of previous abdominal surgery was preferentially included in the 2-day CP group.

Inclusion and exclusion criteria for $\mathrm{CP}$ was summarized in Table 1.

One- and 2-day CPs had the same preoperative and postoperative orders. Lists of IV and PO medications were the same between two CPs.

All patients admitted via outpatient clinic and received elective laparoscopic cholecystectomy. The operation proceeded according to conventional laparoscopic cholecystectomy. The number of laparoscopic ports ranged from single port to 3 ports. The choice of port number determined by various factors such as preference of patient, patient's age and BMI, history of previous surgery, severity of comorbidities, among others.

Patients visited outpatient department 1 week after the discharge day and complete blood cell count and liver function follow up tests were obtained. After 1 month from the first follow up visit, a second outpatient follow up visit was done. Final postoperative complications data was analyzed including medical records of outpatient clinic.

Numerical Rating Scale (NRS) pain scores were checked during the admission at 8-hour intervals and the number of injection of analgesics was counted.

Total admission cost and actual patient's expenditures were also analyzed. The actual expenditure of the patient was calculated as the total medical expenses minus the national health insurance payments.

Statistical analysis was done by SPSS version 22 (IBM: Armonk, NY, USA). Heterogeneity of characteristics of two groups was determined by Pearson ${ }^{2}{ }^{2}$ test (in case of comparison of proportion) and Student t-test (in case of comparison of continuous variables) with significance set at $p=0.05$.

\section{RESULTS}

From March 2016 to October 2016, among 241 patients who underwent elective laparoscopic cholecystectomy.

With exclusion of 170 patients, finally 71 were enrolled and analyzed. Selected patients were admitted through outpatient department and 1-day CP or 2-day CP was applied, based on

Table 1. Inclusion and exclusion criteria for laparoscopic cholecystectomy CP

\begin{tabular}{ll}
\multicolumn{1}{c}{ Inclusion criteria } & \multicolumn{1}{c}{ Exclusion criteria } \\
\hline Gallbladder stone, gallbladder polyp & Cholecystitis with complications (empyema, perforation) \\
Admitted via out-patient department & Accompanied CBD stone \\
Agreed with critical pathway & Emergency operation \\
& ASA classification of 3 or more \\
& Open conversion \\
& Operative complication; biliary tract injury \\
& Refused to be discharged as scheduled \\
& Disagreed with critical pathway \\
\hline
\end{tabular}

ASA $=$ American Society of Anesthesiologists; $C B D=$ common bile duct . 


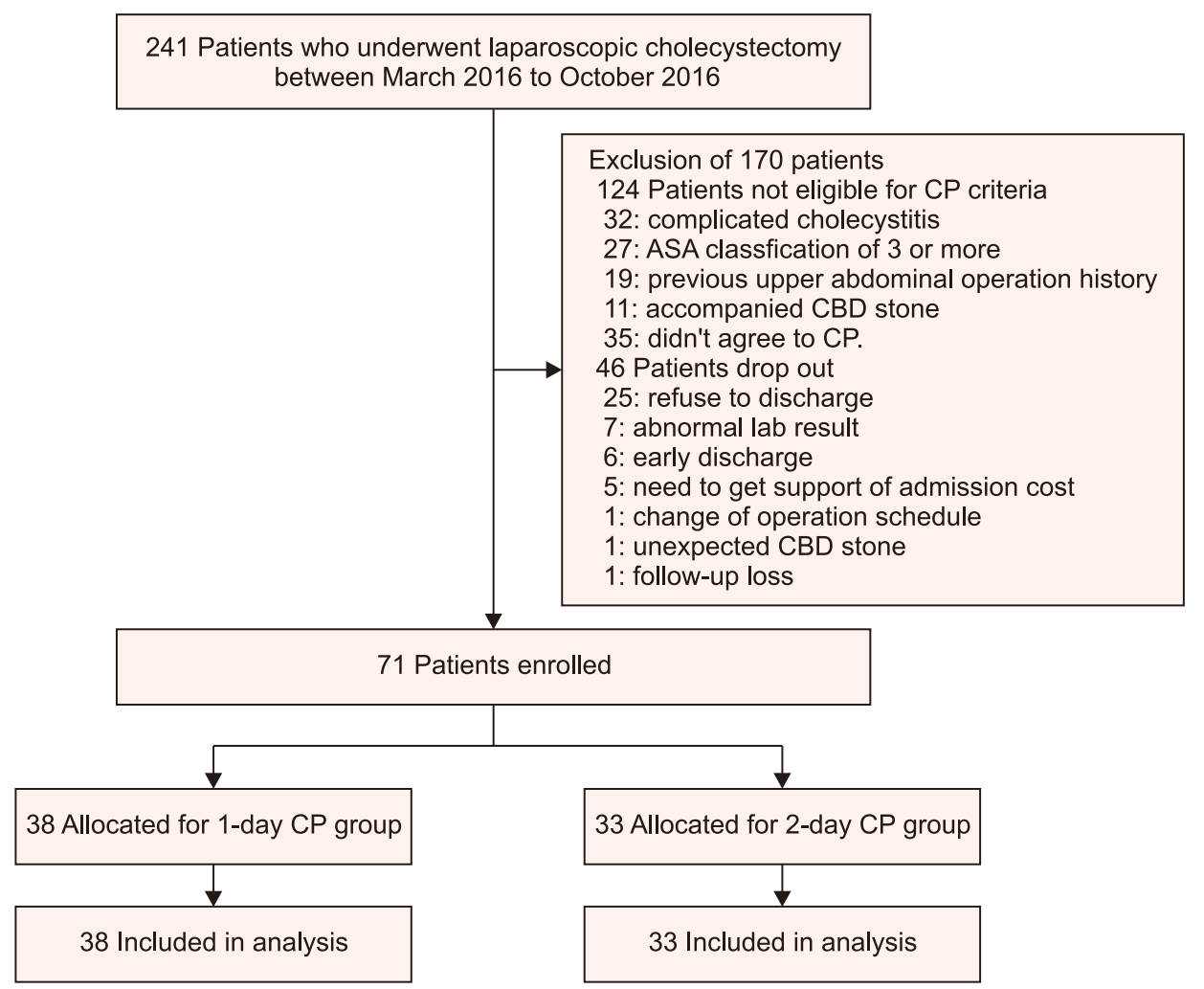

Fig. 1. Patients' flow chart.

patient's characteristics. Patients were divided into two groups for analysis, 38 patients in the 1-day CP group and 33 patients in the 2-day CP group.

Among 170 excluded patients, 124 were not eligible for CP. Most common reasons for ineligibility were complicated cholecystitis (32 cases) such as gallbladder empyema or perforation. Twenty-seven patients with ASA (American Society of Anesthesiologists) classification of 3 or more were excluded. Nineteen patients had previous upper abdominal operation history and 11 concomitant common bile duct stone. Finally, 35 patients did not agree to be enrolled in CP.

Forty-six patients were dropped out during admission. The most common reason was refusal to be discharged ( 25 cases) followed by abnormal laboratory results ( 7 cases), earlier discharge ( 6 cases), need to get financial support (5 cases), change of operation schedule (1 case), and unexpected concomitant common bile duct stone (1 case). One patient was lost during follow up (Fig. 1).

Pre-operative characteristics of 2-day CP and 1-day CP groups are shown in Table 2. Among the list, there was no statistically significant difference between two groups in age (52 vs 49 years old, $p=0.472$ ), patients with comorbidities (51.5\% vs $44.8 \%, p=0.127$ ), abdominal surgery history ( $27.3 \%$ vs $18.4 \%$, $p=0.373$, pre-operative endoscopic retrograde cholangiopancreatography (ERCP) $(15.2 \%$ vs $13.2 \%, p=0.810)$, and pathologic diagnosis $(p=0.757)$.
Operative outcomes were summarized in Table 3. Two-day $\mathrm{CP}$ group showed longer operative time than 1 -day $\mathrm{CP}$ group (73.4 vs $54.1 \mathrm{~min}, p<0.001$ ); otherwise, there was no significant difference among other postoperative outcomes. Postoperative complications observed were 1 surgical site infection and 1 liver abscess in 2-day CP group, and 1 surgical site infection in 1-day CP group. There was no significant difference between 2-day $\mathrm{CP}$ and 1-day $\mathrm{CP}$ in the frequency of complications $(6.1 \%$ vs $2.6 \%, p=0.474)$, NRS pain scale $(1.82$ vs 2.16 , $p=0.052$ ), count of analgesics injection ( 0.12 vs $0.16, p=0.754$ ), outpatient follow-up laboratory values (WBC count; 6494 vs 6724/mm $\mathrm{m}^{3}, p=0.512$, Hemoglobin; 13.5 vs $13.7 \mathrm{~g} / \mathrm{dl}, p=0.604$, Total bilirubin; 0.54 vs $0.54 \mathrm{mg} / \mathrm{dl}, p=0.995$, AST; 18.3 vs 21.5 IU/L, $p=0.137$, ALT; 21.8 vs 27.7 IU/L, $p=0.208$ ), total admission cost ( 347.04 vs $306.69 \times 10^{4}$ won, $\left.p=0.106\right)$, actual patient's expenditures ( 147.85 vs $125.58 \times 10^{4}$ won, $p=0.276$ ).

\section{DISCUSSION}

Recently, the application of critical pathway to laparoscopic cholecystectomy has been steadily increasing because it is associated with shorter hospital stay, reduced hospital costs and increased availability of hospital bed capacity. ${ }^{3,10,11}$

However, there are still some studies reporting unrecognized complications during laparoscopic cholecystectomy, especially in elderly patients with multiple comorbidities. ${ }^{12,13}$ It is a rea- 
Table 2. Patients' preoperative characteristics comparing 2-day CP and 1-day CP

\begin{tabular}{|c|c|c|c|c|}
\hline & All population $(\mathrm{N}=71)$ & 2-day CP (N=33) & 1-day CP (N=38) & $p$ value \\
\hline Age (years) & & & & 0.472 \\
\hline Mean (SD) & $50(14)$ & $52(13)$ & $49(15)$ & \\
\hline Median (range) & $48(24 \sim 81)$ & $50(31 \sim 78)$ & $46(24 \sim 81)$ & \\
\hline Gender, N $(\%)$ & & & & 0.295 \\
\hline Male & $37(52.1)$ & $15(45.5)$ & $22(57.9)$ & \\
\hline Female & $34(47.9)$ & $18(55.5)$ & $16(42.1)$ & \\
\hline Comorbidity*, N $(\%)$ & & & & 0.127 \\
\hline Multiple comorbidities & $9(12.7)$ & $7(21.2)$ & $2(5.3)$ & \\
\hline One comorbidity & $25(35.2)$ & $10(30.3)$ & $15(39.5)$ & \\
\hline None & $37(52.1)$ & $16(48.5)$ & $21(55.3)$ & \\
\hline Abdominal surgery, $\mathrm{N}(\%)$ & & & & 0.373 \\
\hline Yes & $16(22.5)$ & $9(27.3)$ & $7(18.4)$ & \\
\hline No & $55(77.5)$ & $24(72.7)$ & $31(81.6)$ & \\
\hline Pre-op ERCP, N $(\%)$ & & & & 0.810 \\
\hline Yes & $10(14.1)$ & $5(15.2)$ & $5(13.2)$ & \\
\hline No & $61(85.9)$ & $28(84.8)$ & $33(86.8)$ & \\
\hline Diagnosis (pathologic), N (\%) & & & & 0.757 \\
\hline Cholecystitis without stone & $22(31.0)$ & $9(27.3)$ & $13(34.2)$ & \\
\hline Cholecystitis with stone & $36(50.7)$ & $18(54.5)$ & $18(47.4)$ & \\
\hline Gallbladder polyp & $8(11.3)$ & $3(9.1)$ & $5(13.2)$ & \\
\hline Porcelain gallbladder & $1(1.4)$ & $1(3.0)$ & $0(0.0)$ & \\
\hline Xanthogranulomatosis & $4(5.6)$ & $2(6.1)$ & $2(5.3)$ & \\
\hline
\end{tabular}

$\mathrm{CP}=$ critical pathway; $\mathrm{SD}=$ standard deviation; ERCP = endoscopic retrograde cholangiopancreatography. ${ }^{*}$ Including diabetes mellitus, hypertension, cerebrovascular accident, angina and systemic diseases regarded in ASA classification.

sonable concern laparoscopic cholecystectomy using critical pathway may neglect some of the later complications because of forced earlier hospital discharge. Furthermore, complications such as bile duct injury, hemobilia and bowel injury could be life-threatening unless timely detected and properly treated. This is the reason why the critical pathway could not be applied collectively to all patients. In this study, among 241 elective cholecystectomy patients critical pathway was applied to only 117 patients (48.5\%). Our critical pathway inclusion rate is lower than other reports because we were more conservative in including patients to critical pathway.

During hospitalization, 46 cases of drop-out were found, however, there was no case of drop-out related to postoperative complications, and none had different treatment or intervention during admission. Most of the drop-out cases were refusal to be discharged complaining postoperative abdominal pain or discomfort. All of them were discharged after one or two more days of admission without different treatment than usual.

There was significant difference in terms of operative time between 1-day and 2-day CP groups. Since the decision to include either in 1-day or 2-day group was taken by the surgeon's preference or at the patients' request, selection bias was inevitable. Although not statistically significant, preoperative characteristics (Table 2) showed patients with multiple comorbidities and history of previous abdominal surgery was preferentially included in the 2-day $\mathrm{CP}$ group, this factor also may have affected in operative time.

Except for two superficial surgical site infections, there was only one actual postoperative complication in this study. A patient who completed 2-day CP, returned to hospital to outpatient clinic six days after discharge with fever and chilling 
Table 3. Comparison of operative outcomes of 2-day CP and 1-day CP

\begin{tabular}{|c|c|c|c|c|}
\hline & All population $(\mathrm{N}=71)$ & 2-day $\mathrm{CP}(\mathrm{N}=33)$ & 1-day $\mathrm{CP}(\mathrm{N}=38)$ & $p$ value \\
\hline Port number, $\mathrm{N}(\%)$ & & & & 0.084 \\
\hline Single port & $31(43.7)$ & $18|54.6|$ & $13(34.2)$ & \\
\hline Multiport & $40(56.3)$ & $15(45.4)$ & $25(65.8)$ & \\
\hline Operation time (min) & & & & $<0.001$ \\
\hline Mean (SD) & $63.1(23.5)$ & $73.4(24.7)$ & $54.1(18.4)$ & \\
\hline Median (range) & $59(25 \sim 125)$ & $70(40 \sim 125)$ & $53(25 \sim 96)$ & \\
\hline Complication, N $(\%)$ & & & & 0.474 \\
\hline Yes & $3(4.2)$ & $2(6.1)$ & $1(2.6)$ & \\
\hline No & 68 (95.8) & 31 (93.9) & $37(97.4)$ & \\
\hline Pain scale, NRS & & & & 0.052 \\
\hline Mean (SD) & $2.00(0.74)$ & $1.82(0.64)$ & $2.16(0.79)$ & \\
\hline Median (range) & $2(0 \sim 3)$ & $2(0 \sim 3)$ & $2(0 \sim 3)$ & \\
\hline Count of analgesics injection, $\mathrm{N}$ & & & & 0.754 \\
\hline Mean (SD) & $0.14(0.49)$ & $0.12(0.42)$ & $0.16(0.55)$ & \\
\hline Median (range) & $0(0 \sim 3)$ & $0(0 \sim 2)$ & $0(0 \sim 3)$ & \\
\hline \multicolumn{5}{|l|}{$\begin{array}{l}\text { Outpatient follow-up laboratory } \\
\text { values, Mean (SD) }\end{array}$} \\
\hline WBC $\left(/ \mathrm{mm}^{3}\right)$ & $6617(1458)$ & 6494 (1537) & 6724 (1397) & 0.512 \\
\hline Hemoglobin $(\mathrm{g} / \mathrm{d})$ & $13.6(1.2)$ & $13.5(1.1)$ & $13.7(1.4)$ & 0.604 \\
\hline Total bilirubin (mg/dl) & $0.54(0.32)$ & $0.54(0.41)$ & $0.54(0.24)$ & 0.995 \\
\hline AST (IU/L) & $20.0(9.2)$ & $18.3(5.4)$ & $21.5(11.4)$ & 0.137 \\
\hline ALT (IU/L) & $24.9(20.5)$ & $21.8(12.4)$ & $27.7(25.4)$ & 0.208 \\
\hline Total admission cost, $\times 10^{4}$ won & & & & 0.106 \\
\hline Mean (SD) & $331.51(34.23)$ & 347.04 (25.95) & 306.69 (29.80) & \\
\hline Median (range) & 328.39 (267.85 398.47) & 343.95 (281.55 398.47) & $296.38(267.85 \sim 385.00)$ & \\
\hline $\begin{array}{l}\text { Actual patient's expenditure, } \\
\times 10^{4} \text { won }\end{array}$ & & & & 0.276 \\
\hline Mean (SD) & $138.03(24.82)$ & $147.85(20.67)$ & $125.58(27.97)$ & \\
\hline Median (range) & $137.04(77.47 \sim 179.66)$ & 150.74 (89.86 189.66) & 122.66 (77.47 185.64) & \\
\hline
\end{tabular}

sensation. Multiple sub centimeter-sized multiple liver $a b^{-}$ scesses were found on abdominal CT scan. Since this patient had preoperative history of common bile duct stone removal via ERCP, we presumed that subclinical cholangitis existed preoperatively, which developed into multiple small liver abscesses. He was readmitted and properly treated with IV antibiotics and discharged uneventfully on seventh hospital day.

We could not identify statistically significant difference of medical cost between 1-day and 2-day CP groups in this study. However, 1-day CP group showed lower mean and me- dian value of medical cost than 2-day CP group as to both total hospital costs and patients' expenditures.

Recently, same-day discharge CP after laparoscopic cholecystectomy has been adopted in some hospitals. Henri et al. ${ }^{14}$ found that careful patient selection, patient education, and experienced surgical team were needed for successful outpatient laparoscopic cholecystectomy utilizing critical pathway. Thomas et al. ${ }^{15}$ reported that outpatient laparoscopic cholecystectomy with critical pathway was not successful in terms of postoperative recovery in patients older than 50 years, ASA 
score of 3 or more, and those operated after $1 \mathrm{pm}$. Discharge plan scheduled in critical pathway was delayed in those patients group.

We did not apply same-day discharge CP of laparoscopic cholecystectomy in our hospital, because we were not sure about the benefits of this kind of patient management strategy and also, there is no firm conclusion whether sameday discharge is safe or not. Beside, our patient population is generally reticent about being discharged on the same day of surgery. Also, in surgical innovation, we thought that conservative step-by-step adaptation is an important policy.

In this study, we did not impose limitation on patients' age or scheduled operation time for CP enrollment. Actually, oldest patient who underwent laparoscopic cholecystectomy with $\mathrm{CP}$ was 81 years old and completed 1-day CP without any complications and there was no case of drop out from $\mathrm{CP}$ for afternoon operation schedule. Based on this study, we thought further study about feasibility and safety of outpatient laparoscopic cholecystectomy with $\mathrm{CP}$ in selected patients is possible.

In conclusion, though this study has a limitation of being a retrospective review with possible selection bias, it is not only feasible and safe, but also practical the application of 1-day CP for selected patients who undergo laparoscopic cholecystectomy. Our message is 1-day CP application is not inferior to 2-day CP and we hope this study would be helpful for surgeons who wishes to reduce postoperative hospital stay after laparoscopic cholecystectomies.

Nevertheless, larger prospective randomized comparative trials are necessary to confirm our affirmations and draw definitive conclusions.

\section{CONFLICT OF INTEREST}

None.

\section{FUNDING}

None.

\section{ACKNOWLEDGMENTS}

None.

\section{REFERENCES}

1) McMahon AJ, Fischbacher CM, Frame SH, MacLeod MC. Impact of laparoscopic cholecystectomy: a population-based study. Lancet 2000;356:1632-1637.

2) Shea JA, Berlin JA, Bachwich DR, et al. Indications for and outcomes of cholecystectomy: a comparison of the pre and postlaparoscopic eras. Ann Surg 1998;227:343-350.

3) Topal B, Peeters G, Verbert A, Penninckx F. Outpatient laparoscopic cholecystectomy: clinical pathway implementation is efficient and cost effective and increases hospital bed capacity. Surg Endosc 2007;21:1142-1146.

4) Keus F, de Jong JA, Gooszen HG, van Laarhoven CJ. Laparoscopic versus open cholecystectomy for patients with symptomatic cholecystolithiasis. Cochrane Database Syst Rev 2006:Cd006231.

5) Briggs CD, Irving GB, Mann CD, et al. Introduction of a day-case laparoscopic cholecystectomy service in the UK: a critical analysis of factors influencing same-day discharge and contact with primary care providers. Ann R Coll Surg Engl 2009;91:583-590.

6) Tenconi SM, Boni L, Colombo EM, Dionigi G, Rovera F, Cassinotti E. Laparoscopic cholecystectomy as day-surgery procedure: current indications and patients' selection. Int J Surg 2008;6 Suppl 1: S86-88.

7) Vaughan J, Gurusamy KS, Davidson BR. Day-surgery versus overnight stay surgery for laparoscopic cholecystectomy. Cochrane Database Syst Rev 2013:Cd006798.

8) Kehlet H. Multimodal approach to control postoperative pathophysiology and rehabilitation. Br J Anaesth 1997;78:606-617.

9) Kehlet H, Wilmore DW. Evidence-based surgical care and the evolution of fast-track surgery. Ann Surg 2008;248:189-198.

10) Calland JF, Tanaka K, Foley E, et al. Outpatient laparoscopic cholecystectomy: patient outcomes after implementation of a clinical pathway. Ann Surg 2001;233:704-715.

11) Zhang M, Zhou SY, Xing MY, Xu J, Shi XX, Zheng SS. The application of clinical pathways in laparoscopic cholecystectomy. Hepatobiliary Pancreat Dis Int 2014;13:348-353.

12) Alrajraji M, Nawawi A, Jamjoom R, Qari Y, Aljiffry M. Delayed hemobilia due to hepatic artery pseudo-aneurysm: a pitfall of laparoscopic cholecystectomy. BMC Surg 2016;16:59.

13) Little M, Munipalle PC, Nugud O. A rare late complication of laparoscopic cholecystectomy. BMJ Case Rep 2013;2013.

14) Vuilleumier H, Halkic N. Laparoscopic cholecystectomy as a day surgery procedure: implementation and audit of 136 consecutive cases in a university hospital. World J Surg 2004;28:737-740.

15) Robinson TN, Biffl WL, Moore EE, Heimbach JK, Calkins CM, Burch JM. Predicting failure of outpatient laparoscopic cholecystectomy. Am J Surg 2002;184:515-518; discussion 518-519. 